\title{
Transplantation of Neural Stem Cells Cultured in Alginate Scaffold for Spinal Cord Injury in Rats
}

\author{
Seyed Mojtaba Hosseini ${ }^{1-4}$, Ali Sharafkhah ${ }^{1,2}$, Omid Koohi-Hosseinabadi ${ }^{1,2,5}$, Maryam Semsar-Kazerooni ${ }^{1,2}$ \\ ${ }^{1}$ Student Research Committee, Shiraz University of Medical Sciences, Shiraz, Iran \\ ${ }^{2}$ Cell and Molecular Medicine Student Research Group, Medical Faculty, Shiraz University of Medical Sciences, Shiraz, Iran \\ ${ }^{3}$ Shiraz Neuroscience Research Center, Shiraz University of Medical Sciences, Shiraz, Iran \\ ${ }^{4}$ Stem Cell Laboratory, Department of Anatomy, Shiraz University of Medical Sciences, Shiraz, Iran \\ ${ }^{5}$ Laboratory Animals Center, Shiraz University of Medical Sciences, Shiraz, Iran
}

Study Design: This study investigated the effects of transplantation of alginate encapsulated neural stem cells (NSCs) on spinal cord injury in Sprague-Dawley male rats. The neurological functions were assessed for 6 weeks after transplantation along with a histological study and measurement of caspase-3 levels.

Purpose: The aim of this study was to discover whether NSCs cultured in alginate transplantation improve recovery from spinal cord injury.

Overview of Literature: Spinal cord injury is one of the leading causes of disability and it has no effective treatment. Spinal cord injury can also cause sensory impairment. With an impetus on using stem cells therapy in various central nervous system settings, there is an interest in using stem cells for addressing spinal cord injury. Neural stem cell is one type of stem cells that is able to differentiate to all three neural lineages and it shows promise in spinal injury treatment. Furthermore, a number of studies have shown that culturing NSCs in three-dimensional (3D) scaffolds like alginate could enhance neural differentiation.

Methods: The NSCs were isolated from 14-day-old rat embryos. The isolated NSCs were cultured in growth media containing basic fibroblast growth factor and endothelial growth factor. The cells were characterized by differentiating to three neural lineages and they were cultured in an alginate scaffold. After 7 days the cells were encapsulated and transplanted in a rat model of spinal cord injury. Results: Our data showed that culturing in an alginate 3D scaffold and transplantation of the NSCs could improve neurological outcome in a rat model of spinal cord injury. The inflammation scores and lesion sizes and also the activity of caspase-3 (for apoptosis evaluation) were less in encapsulated neural stem cell transplantation cases.

Conclusions: Transplantation of NSCs that were cultured in an alginate scaffold led to a better clinical and histological outcome for recovery from spinal cord injury in a rat model.

Keywords: Neural stem cells; Alginate; Spinal cord injuries

\section{Introduction}

Spinal cord injury is a significant condition of unmet medical need and its prevalence is rising each year. The mortality from spinal cord injury has remained at $5 \%$ for many years; however, the disability from this condition is a major concern of the clinicians and patients around the world [1]. Contusions and compressions are the most

Received Oct 2, 2015; Revised Oct 21, 2015; Accepted Oct 21, 2015

Corresponding author: Seyed Mojtaba Hosseini

Stem Cell Laboratory, Department of Anatomy, Medical Faculty,

Shiraz University of Medical Sciences, Zand Blvd, Shiraz, Iran

Tel: +98-71-32122970, Fax: +98-71-32122970, E-mail: Hoseini2010m@gmail.com 
common types of spinal cord injury that could physically damage the spinal cord followed by formation of a glial scar, demyelization and axonal damage [2-4]. Spinal cord injury pathophysiology has two steps: first, the primary damage caused by the mechanical contusion and second, the secondary damage that caused by inflammation, axonal damage and increasing numbers of astroglia $[5,6]$. Currently, there is no treatment for spinal cord injury and the rate of spontaneous neurogenesis is very low in the patients $[6,7]$.

Cell therapy is a new approach to neurodegenerative diseases exploring the utility of neural stem cells present in adult and fetus central nervous system (CNS) where they are capable of differentiating to three neural lineages cells including neurons, oligodendrocytes and astrocytes [8-10]. The neural stem cells are usually described as undifferentiated cells with the ability to self renewal and capacity to proliferate and produce new nervous tissue [11]. Also the neural stem cells might have neuroprotective effects and reduce inflammation via peroxisomeproliferator-activated receptor [12] and secrete necessary cytokines and express characteristic integrins $[11,13]$.

Therefore, due to the neural stem cells properties mentioned above, such cells could be an appropriate choice for cell therapy in spinal cord injury model. Banerjee et al. [14] in 2009, demonstrated that an elastic modulus of alginate three-dimensional (3D) which was comparable to brain tissue elasticity could enhance the neural differentiation of neural stem cells. An alginate scaffold could increase neural differentiation of the other stem cells type such as induced pluripotent stem cells [15].

In this study, we decided to investigate the effects of transplantation of alginate encapsulated neural stem cells on spinal cord injury in a spinal cord injury model in rats and compare it with effects of 2D cultured neural stem cells post transplantation.

\section{Materials and Methods}

\section{Experimental design}

Sixty Sprague-Dawley male rats weighting 250-300 g were randomly selected for this study and they were divided to four groups $(\mathrm{n}=15)$ as below: (1) Control group: this group received no intervention and no treatment. (2) Sham group: spinal cord injury was induced in this group with no subsequent treatment given. (3) Neural stem cell transplantation group: this group received neural stem cells locally 1 day after spinal cord injury. (4) Alginate encapsulated neural stem cells transplantation: this group received neural stem cells cultured in alginate scaffold 1 day after spinal cord injury.

\section{Animal preparation}

All procedures in this study were approved by Shiraz University of Medical Sciences Ethical Committee.

The animals had free access to water and food.

\section{Isolation and expansion of neural stem cells}

The neural stem cells were isolated from the ganglion eminence of a 14-day embryo of Sprague-Dawley rat. Briefly, the ganglion eminence of a rat embryo was dissected and after pipetting the neural stem cells into culture media (DMEM/F12, B27 2\%, basic fibroblast growth factor $10 \mathrm{ng} / \mathrm{mL}$, endothelial growth factor $20 \mathrm{ng} / \mathrm{mL}$ and penicillin/streptomycin at $1 \%$ ), they were transferred to T-25 $\mathrm{cm}^{2}$ flasks and incubated in a humidified $37^{\circ} \mathrm{C}, 5 \%$ $\mathrm{CO}_{2}$ incubator for 5 days, after which spheres had formed in the media which have been referred to as neurospheres. The neurospheres were passaged by adding and washing off trypsin to $0.05 \%$ (catalogue number 07910; Stem Cell Tech, Vancouver, BC, Canada) and they were cultured in two culture flasks.

\section{Characterization of cultured neural stem cells}

For confirming the stemness of the isolated cells, their capability to differentiate to three neural lineages (neurons, oligodendrocytes and astrocytes) need to be determined. For differentiating the isolated cells, $5 \%$ fetal bovine serum was added to the culture media. After 3 days, the neural stem cells differentiated to three neural cell types as was assessed with immunocytochemistry of microtubuleassociated protein 2 (MAP-2) for detecting neurons and glial fibrillary acidic protein (GFAP) for astrocytes (the method described below).

\section{Immunocytochemistry for neural stem cells}

To assess the differentiation of neural stem cells to neurons and astrocytes the immunocytochemistry was performed with MAP-2 antibody (for detecting neurons) and 
GFAP (for detecting astrocytes). Briefly, the cells were fixed with paraformaldehyde $4 \%$ at $4^{\circ} \mathrm{C}$ for 20 minutes, followed by washing with phosphate buffer saline (PBS). The primary antibodies for MAP-2 (Abcam, Cambridge, UK; ab5392 1:1000) and GFAP (DAKO, Glostrup, Denmark; catalogue number Z0334 1:700) in 5\% goat serum and $0.01 \%$ Triton-X100 were added and the cells were kept at room temperature for 1 hour. The primary antibodies were then removed and the cells were twice washed with PBS and the secondary antibodies were added for 1 hour in room temperature. For final step, the cells were washed three times with PBS and after that visualized and photographed under a fluorescent microscope (model Nikon X66; Nikon, Tokyo, Japan).

\section{4', 6-diamino-2-phenylindole dihydrochloride staining}

For detecting cell nuclei, the cells were fixed with paraformaldehyde $4 \%$ at $4^{\circ} \mathrm{C}$ for 20 minutes. After that the 4', 6-Diamino-2-phenylindole dihydrochloride (DAPI) (Millipore, Billerica, MA, USA; catalogue number 124653) was added $(1: 1,000)$ and the cells were kept for 30 minutes at room temperature.

\section{Neural stem cell culture in alginate scaffold}

Neural stem cells were cultured in alginate (SigmaAldrich, St. Louis, MO, USA) scaffold for 7 days at a concentration of 4,000 cells/well. The sterile sodium alginate was dissolved in DMEM/F12 (alginate concentration $0.25 \%$ ) and resuspended with 4,000 cells in $40 \mu \mathrm{L}$. The solution was then transferred to 96-well plates and $100 \mu \mathrm{L}$ of chilled $\mathrm{CaCl}_{2}$ (Sigma-Aldrich) at $10 \mathrm{mM}$ was added, and the cells were kept on ice for 10 minutes. Subsequently, 50 $\mu \mathrm{L}$ of the suspension were removed and the neural stem cells culture media and laminin at $5 \mu \mathrm{g} / \mathrm{mL}$ was added to each well. For releasing the encapsulated neural stem cells, the scaffold was washed with PBS and a solution containing $15 \mathrm{mM}$ sodium citrate and $150 \mathrm{~m} \mathrm{MNaCl}$ (SigmaAldrich) was added and the cells were incubated in $37^{\circ} \mathrm{C}$ for 1 hour [14].

\section{Neural stem cell transplantation}

The cultured neural stem cells were resuspended in complete media at a concentration of $10^{6}$ cells $/ 100 \mu \mathrm{L}$. Each rat received $10 \mu \mathrm{L}$ of the mentioned suspension $\left(10^{5}\right.$ cells $)$ that was injected by a Hamilton syringe (Sigma-Aldrich) in 20 minutes [16]. 100,000 cells cultured in the 3D scaffold were transplanted to the intra-lesion 1 day after the spinal cord injury.

\section{Surgical intervention}

The aneurysm clip model of spinal cord injury was used in this study. With the animals under general anesthesia with halothane and $\mathrm{O}_{2} / \mathrm{NO}_{2}$ mixture (1:1), a midline incision in the level of T4-T9 was made and the connective tissues were dissected and laminectomy was performed in the area of T6-T8. The rats received the clip at $23 \mathrm{~g}$ for 1 minute at the level of T7 to induce spinal cord injury. After 1 minute the wounds were sutured and the rats were kept at temperature of $27^{\circ} \mathrm{C}$ and their bladders were expressed manually three times a day [17].

\section{Basso, Beattie, and Bresnahan open-field locomo- tion scoring}

For examining the rats' motor performance the Basso, Beattie, and Bresnahan (BBB) scoring was used twice a week for each rat by an examiner blinded to the rat's treatment. The BBB score (with a scale of 0 to 21) was used to evaluate the hind-limb locomotors recovery containing joint movement, stepping ability, trunk stability and coordination. A score of 21 indicated no impairment as seen in uninjured rats [18]. The BBB test was performed twice a week for 12 weeks. The BBB score for the rats after the inducing spinal cord injury model was $8.22 \pm 0.36$ (mean \pm standard deviation $[S D]$ ).

\section{Histology study}

For assessing the necrosis area due to spinal cord injury the cryosections of spinal cord were provided and stained with hematoxylin and eosin. The section intervals were 2 $\mathrm{mm}$ from dorsal to ventral sides. Each section thickness was $20 \mu \mathrm{m}$. The cells with swelling, pyknosis and karyorrhexis nucleus and disrupted cell membrane were marked as necrotic cells. To assess damage quantitatively, the sections were scored from 0 to 3 by a reviewer blinded to the experiment; the presence and intensity of inflammatory cell infiltration, neuronal vacuolation, and hemorrhage was also noted ( 0 was with no evidence and 3 was for a severe infiltration) [19]. 


\section{Apoptosis evaluation with measurement of caspase-3 activity}

Activation of ICE family proteases/caspases begins the pathway of apoptosis in mammalian cells. The apoptosis detection assay used was based on the spectrophotometric detection of chromophore $p$-nitroaniline ( $p$-NA) after cleavage of the labeled substrate DEVD- $p$-NA. The $p$-NA light emission could be measured at $400-405 \mathrm{~nm}$ by a spectrophotometer. The activation of caspase- 3 was assessed 3 days after spinal cord injury. For this evaluation the caspase-3 assay kit from Abcam Company was used (Abcam; cat numberab39401).

\section{Statistical analysis}

All data was reported as mean \pm SD in this study and they were analyzed with one-way analysis of variance test with Graph Pad Prism 6.00 (GraphPad, La Jolla, CA, USA). The significant difference was set at $p$-value $<0.05$.

\section{Results}

\section{Isolation and characterization of rat embryo neural stem cells}

The isolated neural stem cells were cultured as previously described in complete culture media. They formed sphere like cells termed neurospheres after 5 days and also they had a round shape in the alginate 3D culture (Figs. 1, 2).

The cultured neural stem cells differentiated to three

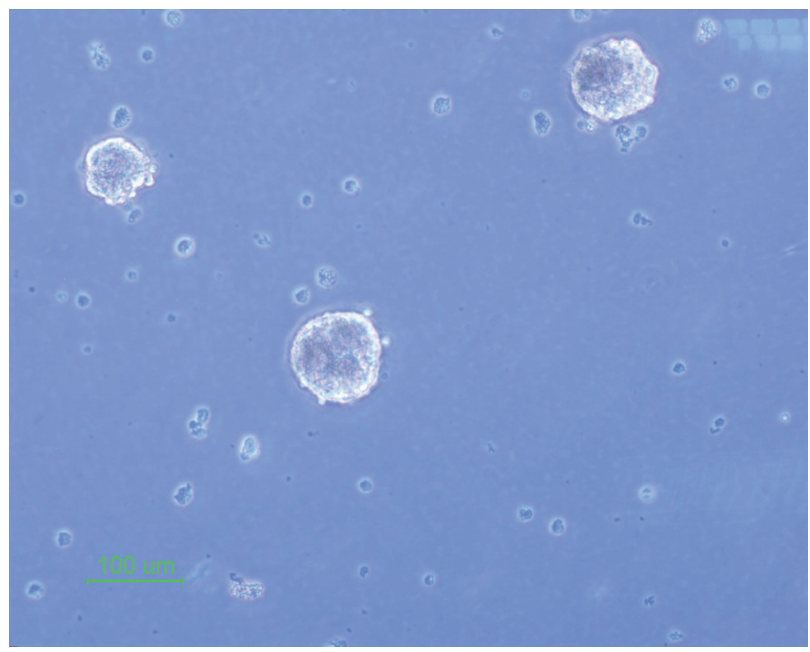

Fig. 1. Neural stem cell culture and neurospheres formation $(\times 10)$. neural lineages 48 hours after adding the fetal bovine serum to the culture medium and they presented different morphologies due to different cell production (neurons, astrocytes and oligodendrocytes) (Fig. 3).

The neural stem cells differentiation to neurons and astrocytes was assessed by immunocytochemistry with MAP-2 and GFAP staining. For MAP-2 (a neuronal marker), $10.46 \% \pm 3.94 \%$ of cells were positive and for GFAP (an astrocyte marker), $89.49 \% \pm 7.40 \%$ of cells were positive (Fig. 4).

\section{Locomotor recovery assessment by BBB scores}

The rats' motor recovery was assessed by $\mathrm{BBB}$ scoring. The $\mathrm{BBB}$ score for the sham group was $8.85 \pm 1.21$ (mean $\pm \mathrm{SD}$ ),

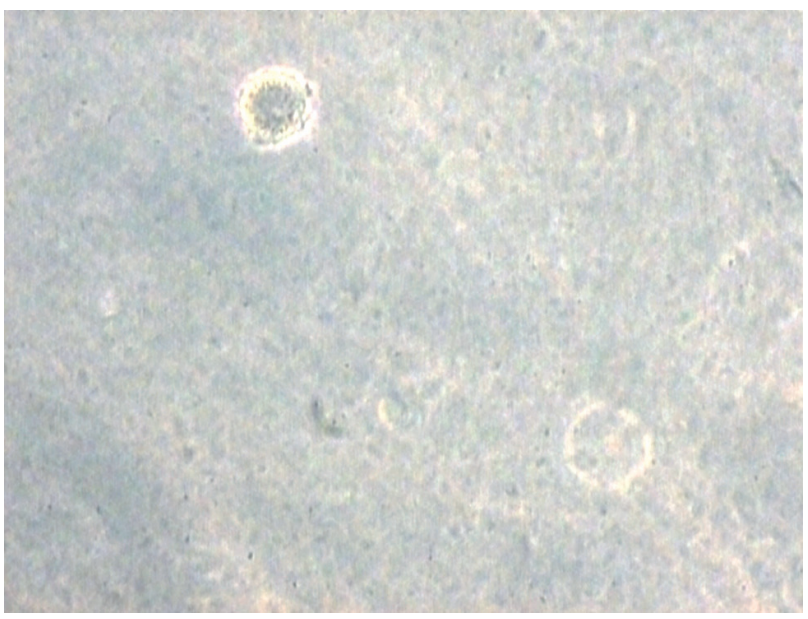

Fig. 2. Neural stem cell culture in alginate scaffold $(\times 40)$.

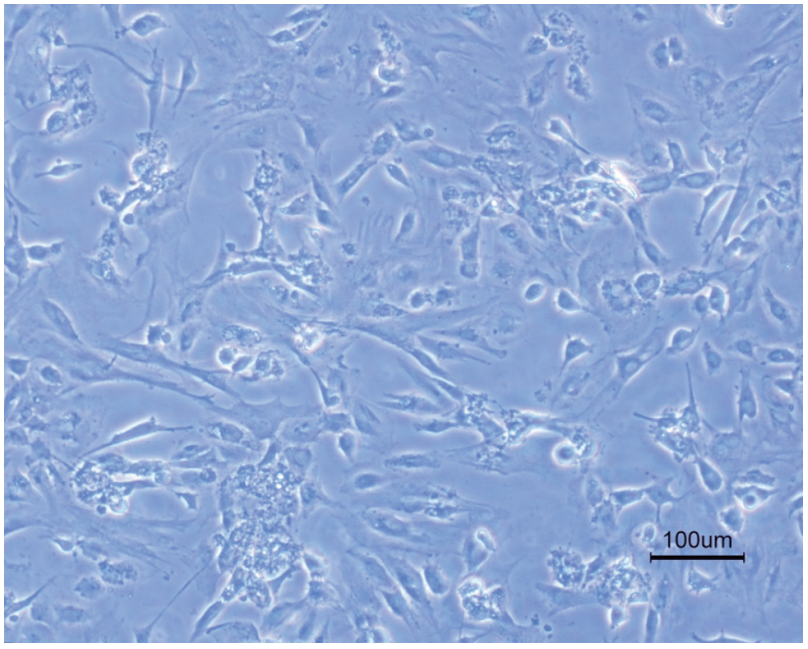

Fig. 3. Neural stem cell differentiation to three neural lineages $(\times 10)$. 


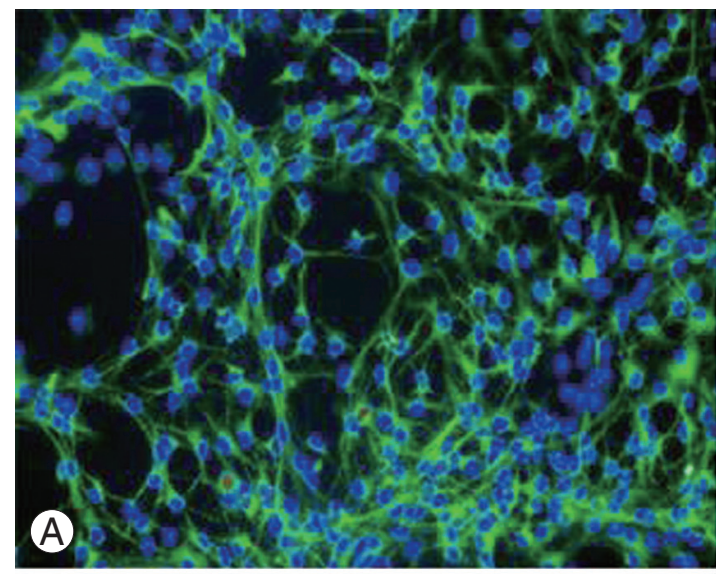

MAP-2-antibody

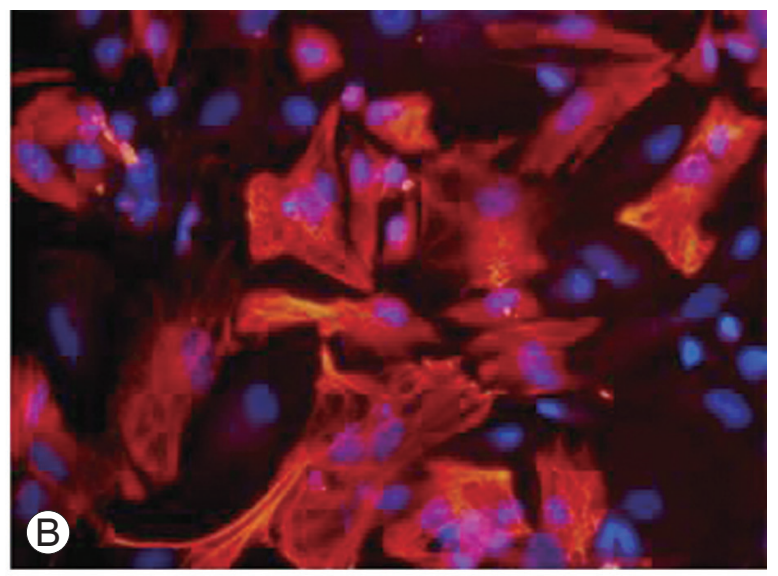

GFAP-antibody

Fig. 4. Immunocytochemistry for neurons and astrocytes. (A) Microtubule-associated protein 2 (MAP-2) immunocytochemistry with Alexa Fluor 488 secondary antibody (×40). (B) Glial fibrillary acidic protein (GFAP) immunocytochemistry with Alexa Fluor 568 secondary antibody $(\times 40)$

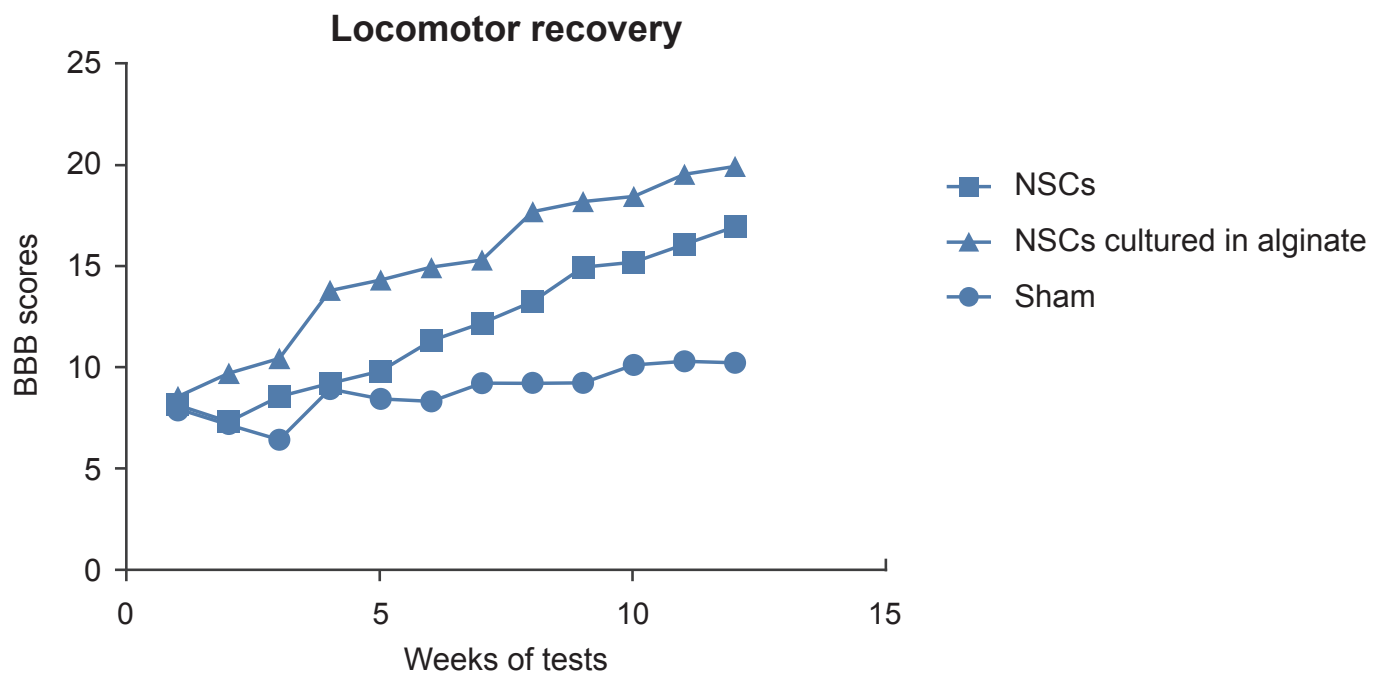

Fig. 5. Basso, Beattie, and Bresnahan (BBB) score. NSCs, neural stem cells.

for the NSCs transplantation group was $11.97 \pm 3.36$ and for NSCs cultured in alginate was $15.13 \pm 3.88$ (Fig. 5). There was a significant difference between each two groups $(p<0.05)$. The data indicated that the recovery in the group which received NSCs cultured in alginate had better recovery than the other two groups.

\section{Histology}

The histology study was based on quantitative scoring as previously described ( $0-3$ scores) [18]. The histology score for the sham group was $2.43 \pm 0.26$, for NSCs transplanted group it was $1.26 \pm 0.24$ and for NSCs cultured in alginate was $0.53 \pm 0.21$. There was a significant difference forthe groups which received NSCs cultured in alginate having a better histology outcome compared to two other groups which matched the better mobility outcome $(p<0.05)$. Also the NSCs transplanted group had better histological readings than the sham group $(p<0.05)$ (Fig. 6).

\section{Apoptosis evaluation by assessing caspase-3 activity}

A higher activity of caspase-3 indicates more apoptosis. The data represents that both groups which received NSCs had less apoptosis than the sham group ( $p$-value less than 0.05 ) and the group that received NSCs cultured in alginate 


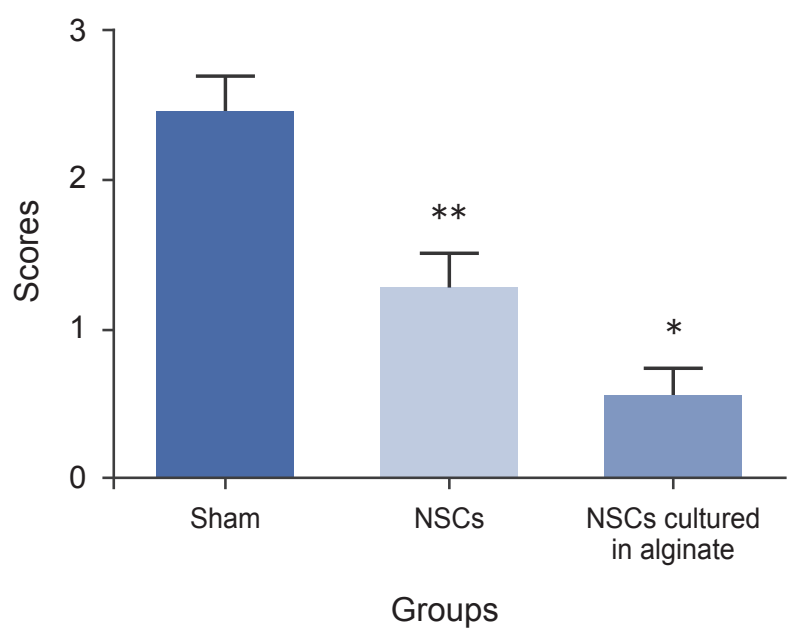

Fig. 6. Histology study. NSCs, neural stem cells. ${ }^{*}$ and ${ }^{* *}$ mean each group has significant difference in comparison with the other groups ( $p$-value $<0.05)$.

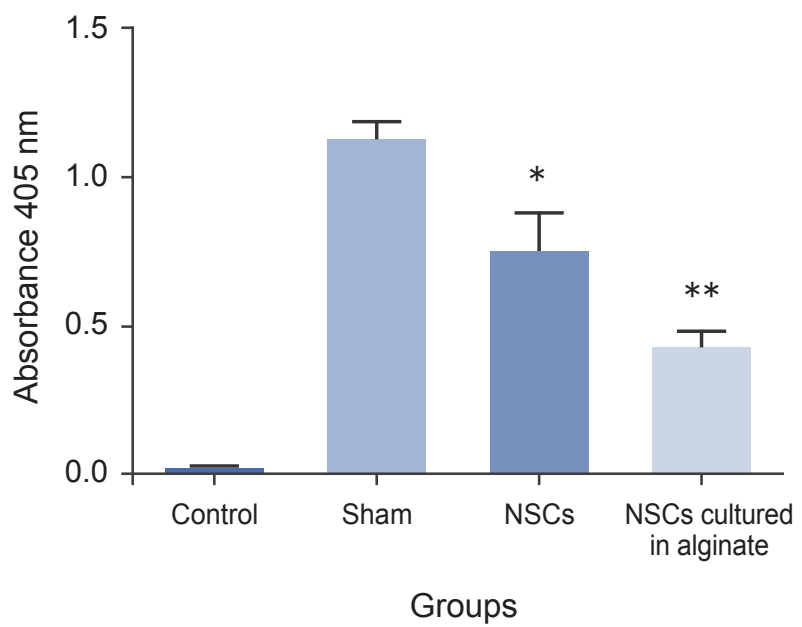

Fig. 7. Caspase-3 activity. NSCs, neural stem cells. ${ }^{*}$ and ${ }^{* *}$ mean each group has significant difference in comparison with the other groups ( $p$-value $<0.05)$.

had the least amount of apoptosis compared to the sham group and the NSCs transplantation group (Fig. 7).

\section{Discussion}

This study presents that cell therapy with neural stem cells could be beneficial for recovery after a spinal cord injury and also that the transplantation of neural stem cells cultured in an alginate scaffold could increase the level of recovery.

Khosravizade et al. [20] showed that the alginate scaffold could enhance neural differentiation of mesenchymal stem cells. Also, Bozza et al. [15] demonstrated that alginate scaffold could promote the neural fates of induced pluripotent stem cells in a three-dimensional culture. In addition, Banerjee et al. [14] indicated that the threedimensional culture of neural stem cells in alginate scaffold improved neural differentiation and neural marker ( $\beta$-tubulin III) expression by neural stem cells. Our previous study showed that the alginate scaffold could provide a microenvironment to differentiate stem cells to motor neurons [21]. In this study, the data shows that the encapsulated neural stem cells in alginate scaffold could be more capable of regeneration in comparison to the twodimensional culture of neural stem cells and this capability could be seen in neurological outcome improvement and also a lower lesion volume from a histological study.

This study has shown that transplantation of alginate encapsulation of neural stem cells could make the apoptosis of spinal cells less severe and also in histological study there was less inflammation in the groups which received neural stem cells as a cell therapy. However, these antiapoptotic and anti-inflammatory effects could be seen to a significantly higher extent with the alginate encapsulated cells. These protective effects form the neural stem cells could be due to the induction of pathogenic apoptosis in Th1 and Th17 T cells [22], and this apoptotic induction might be stronger from alginate encapsulated neural stem cells. Also Kim et al. [23] postulated that the antiinflammatory effects of neural stem cells could be because of reducing interleukin $1 \beta$ (IL-1 $\beta$ ) secretion (as a proinflammatory cytokine) and increasing secretion of IL-10 (as an anti-inflammatory cytokine).

The mechanisms mentioned above have been reported for two-dimensionally cultured neural stem cells but in this study we have shown that the recovery from spinal cord injury was significantly higher for transplanted of alginate encapsulated neural stem cells. This may be because of a higher activity of these mechanisms, although there is a need for more investigation in this area in exploring the mechanisms involved.

\section{Conclusions}

Cell therapy with neural stem cells could be an appropriate approach for treating spinal cord injury and their culture in alginate $3 \mathrm{D}$ scaffold could enhance recovery after transplantation. 


\section{Conflict of Interest}

No potential conflict of interest relevant to this article was reported.

\section{References}

1. Pearse DD, Sanchez AR, Pereira FC, et al. Transplantation of Schwann cells and/or olfactory ensheathing glia into the contused spinal cord: survival, migration, axon association, and functional recovery. Glia 2007;55:976-1000.

2. Dietz V, Curt A. Neurological aspects of spinal-cord repair: promises and challenges. Lancet Neurol 2006; 5:688-94.

3. Kurnellas MP, Nicot A, Shull GE, Elkabes S. Plasma membrane calcium ATPase deficiency causes neuronal pathology in the spinal cord: a potential mechanism for neurodegeneration in multiple sclerosis and spinal cord injury. FASEB J 2005;19:298-300.

4. McDonald JW, Belegu V. Demyelination and remyelination after spinal cord injury. J Neurotrauma 2006; 23:345-59.

5. von Euler M, Seiger A, Sundstrom E. Clip compression injury in the spinal cord: a correlative study of neurological and morphological alterations. Exp Neurol 1997;145:502-10.

6. Pan JZ, Ni L, Sodhi A, Aguanno A, Young W, Hart RP. Cytokine activity contributes to induction of inflammatory cytokine mRNAs in spinal cord following contusion. J Neurosci Res 2002;68:315-22.

7. Sarveazad A, Bakhtiari M, Babahajian A, et al. Comparison of human adipose-derived stem cells and chondroitinase $\mathrm{ABC}$ transplantation on locomotor recovery in the contusion model of spinal cord injury in rats. Iran J Basic Med Sci 2014;17:685-93.

8. Hosseini SM, Farahmandnia M, Razi Z, et al. Combination cell therapy with mesenchymal stem cells and neural stem cells for brain stroke in rats. Int J Stem Cells 2015;8:99-105.

9. Hosseini SM, Talaei-Khozani T, Sani M, Owrangi B. Differentiation of human breast-milk stem cells to neural stem cells and neurons. Neurol Res Int 2014; 2014:807896.

10. Hosseini SM, Samimi N, Farahmandnia M, et al. The preventive effects of neural stem cells and mesenchymal stem cells intra-ventricular injection on brain stroke in rats. N Am J Med Sci 2015;7:390-6.

11. Widera D, Kaus A, Kaltschmidt C, Kaltschmidt B. Neural stem cells, inflammation and NF-kappaB: basic principle of maintenance and repair or origin of brain tumours? J Cell Mol Med 2008;12:459-70.

12. Wang J, Shen Y, Zhang Y, et al. Recent evidence of the regulatory role of PPARs in neural stem cells and their underlying mechanisms for neuroprotective effects. Curr Stem Cell Res Ther 2016;11:188-96.

13. Pluchino S, Zanotti L, Rossi B, et al. Neurosphere-derived multipotent precursors promote neuroprotection by an immunomodulatory mechanism. Nature 2005;436:266-71.

14. Banerjee A, Arha M, Choudhary S, et al. The influence of hydrogel modulus on the proliferation and differentiation of encapsulated neural stem cells. Biomaterials 2009;30:4695-9.

15. Bozza A, Coates EE, Incitti $\mathrm{T}$, et al. Neural differentiation of pluripotent cells in 3D alginate-based cultures. Biomaterials 2014;35:4636-45.

16. Wu S, Cui G, Shao H, Du Z, Ng JC, Peng C. The cotransplantation of olfactory ensheathing cells with bone marrow mesenchymal stem cells exerts antiapoptotic effects in adult rats after spinal cord injury. Stem Cells Int 2015;2015:516215.

17. Rivlin AS, Tator CH. Effect of duration of acute spinal cord compression in a new acute cord injury model in the rat. Surg Neurol 1978;10:38-43.

18. Basso DM, Beattie MS, Bresnahan JC. A sensitive and reliable locomotor rating scale for open field testing in rats. J Neurotrauma 1995;12:1-21.

19. Qiao F, Atkinson C, Kindy MS, et al. The alternative and terminal pathways of complement mediate posttraumatic spinal cord inflammation and injury. Am J Pathol 2010;177:3061-70.

20. Khosravizadeh Z, Razavi S, Bahramian H, Kazemi M. The beneficial effect of encapsulated human adiposederived stem cells in alginate hydrogel on neural differentiation. J Biomed Mater Res B Appl Biomater 2014;102:749-55.

21. Hosseini SM, Vasaghi A, Nakhlparvar N, Roshanravan R, Talaei-Khozani T, Razi Z. Differentiation of Wharton's jelly mesenchymal stem cells into neurons in alginate scaffold. Neural Regen Res 2015;10:13126.

22. Hackett C, Knight J, Mao-Draayer Y. Transplantation of Fas-deficient or wild-type neural stem/progenitor 
cells (NPCs) is equally efficient in treating experimental autoimmune encephalomyelitis (EAE). Am J Transl Res 2014;6:119-28.

23. Kim JA, Ha S, Shin KY, et al. Neural stem cell trans- plantation at critical period improves learning and memory through restoring synaptic impairment in Alzheimer's disease mouse model. Cell Death Dis 2015;6:e1789. 